\title{
Gas Phase Bond Formation in Dipeptide
}

\section{Clusters}

\author{
Maria Nihamkin, ${ }^{\dagger}$ Avinoam Isaak, ${ }^{\ddagger}$ Amnon Albeck, ${ }^{\ddagger}$ Yitzhak Mastai, ${ }^{\top}$ and Yoni \\ Toker $^{*, \dagger}$ \\ $\dagger$ Department of Physics, and Institute of Nanotechnology and Advanced Materials, Bar Ilan \\ University, Ramat Gan 5290002, Israel. \\ $\ddagger$ Department of Chemistry Bar Ilan University, Ramat Gan 5290002, Israel. \\ IDepartment of Chemistry, and Institute of Nanotechnology and Advanced Materials, Bar \\ Ilan University, Ramat Gan 5290002, Israel. \\ E-mail: yonitoker@gmail.com
}

November 13, 2020

\section{Supporting Information}

Dipeptides synthesis: Step A - coupling. To a solution of L-Boc-Ser or D-Boc-Ser (1.0 gr, $4.9 \mathrm{mmol}, 1.0 \mathrm{eq})$ in DCM were added the hydrochloride salt of L-Ser-OMe or D-Ser-OMe (0.83 gr, $5.4 \mathrm{mmol}, 1.1 \mathrm{eq}), \mathrm{Et}_{3} \mathrm{~N}(2 \mathrm{ml}, 14.7 \mathrm{mmol}, 3.0 \mathrm{eq})$ and PyBop reagent (2.8 gr, $5.4 \mathrm{mmol}, 1.1 \mathrm{eq}$ ), and the reaction mixture was stirred at r.t overnight. The DCM was evaporated under reduced pressure, and the residues were dissolved in EtOAc and filtered over a short silica column, washed with excess EtOAc and evaporated. The remaining semisolid was recrystallized from $\mathrm{H}_{2} \mathrm{O} / \mathrm{ACN}$ to give the protected LL-dipeptide (0.85 gr, $57 \%$ ), LD-dipeptide (0.82 gr, 55\%), DL-dipeptide (0.86 gr, 57\%) and DD-dipeptide (0.82 gr, 55\%). 
Step $B$ - deprotection. The protected dipeptides were dissolved in a $5 \mathrm{ml}$ solution of $\mathrm{HCl}$ in dioxane (made from $20 \mathrm{ml}$ of $32 \% \mathrm{HCl}$ solution and $30 \mathrm{ml}$ of dioxane). After 3 days, all the volatiles were evaporated under high vacuum and the remaining semi-solid was recrystallized from $\mathrm{H}_{2} \mathrm{O} / \mathrm{ACN}$ to give the unprotected LL-dipeptide (0.28 gr, 52\%), LD-dipeptide (0.3 gr, $56 \%$ ), DL-dipeptide (0.18 gr, 35\%) and DD-dipeptide (0.31 gr, 57\%).

Tetrapeptide synthesis: Attachment of the first amino acid to trityl chloride resin 0.115 gr of Fmoc-Ser(O-tBu)-OH (0.3 mmol, 1.0 eq) was dissolved in $4 \mathrm{ml}$ of dry DCM. To this solution was added $0.054 \mathrm{ml}$ of DIPEA (0.3 mmol, $1.0 \mathrm{eq})$ and then the solution was pulled up by a $10 \mathrm{ml}$ peptide-synthesis syringe that preloaded with $0.4 \mathrm{gr}$ of trityl chloride resin (loading $0.7,0.3 \mathrm{mmol}, 1.0 \mathrm{eq}$ ). The mixture was agitated for 5 minutes, $0.08 \mathrm{ml}$ of DIPEA (0.45 mmol, $1.5 \mathrm{eq})$ was added, and the agitation continued to another 60 minutes. To endcap any remaining reactive trityl group, $0.24 \mathrm{ml}$ of $\mathrm{MeOH}$ was added and the agitation continued to 15 more minutes. The resin was filtered through the syringe sinter, and then washed three times with DCM, twice with DMF, twice with DCM, three times with methanol and once again with DMF.

Fmoc deprotection - $4 \mathrm{ml}$ of $20 \% \mathrm{v} / \mathrm{v}$ piperidine in DMF solution was pulled up by the reaction syringe and then left to 30 minutes agitation. The resin was filtered through the syringe sinter, and then washed 5 times with DMF and coupled immediately to the next amino acid.

Attachment of the second, third and fourth amino acid - 0.58 gr of Fmoc-Ser(O-tBu)-OH (1.5 mmol, $5.0 \mathrm{eq})$ and $0.53 \mathrm{gr}$ of HBTU (1.5 mmol, $5.0 \mathrm{eq})$ were dissolved in $3 \mathrm{ml}$ of DMF. After complete solvation, $0.27 \mathrm{ml}$ of DIPEA was added and the solution was pulled up by the reaction syringe and agitated for 2 hours. The resin was filtered through the syringe sinter, and then washed once with DMF, three times with i-PrOH, and three time with DCM, alternately, and once again with DMF. The attachment of the third and fourth amino acid was done by repeating the deprotection step and then the coupling.

Final cleavage and side chains deprotection - The Fmoc group was cleaved by the de- 
protection procedure and additionally washed 3 times with diethyl ether and the resin was evaporated to dryness. A solution of $3.8 \mathrm{ml}$ TFA, $0.1 \mathrm{ml}$ water and $0.1 \mathrm{ml}$ triisopropylsilane was pulled up by the reaction syringe and agitated for an overnight period. The solution was injected into a $30 \mathrm{ml}$ ice-cold ether and the precipitate peptide was isolated by rotation in a centrifuge, and washed another three times with ether. The remaining ether was evaporated and the peptide was purified by HPLC.

MSMS measurements - The measurements were conducted using a Water's Q-TOF micromass spectrometer. We have taken care to use the same conditions for each of the measurements. The capillary voltage was set to $3 \mathrm{kV}$, sample cone to $30 \mathrm{~V}$ and the extraction cone set to $4 \mathrm{~V}$. The source temperature was 80 Celsius and the desolvation temperature was 150 Celsius. Argon was used in the collision cell.

Serine octamer fragmentation: We have performed extensive MS/MS measurements on serine clusters and found no evidence for peptide bond formation. Figure 1 shows a summary of the MS/MS results for fragmentation of the magic serine octamer. We show the abundance of the different charged $S_{N} H^{+}$fragments produced by accelerating $S_{8} H^{+}$ clusters into the argon filled collision cell as a function of the collision voltage, $V_{c}$. We normalize by the sum of abundances of all fragments at the particular $V_{c}$. The results indicate (although do not prove conclusively) that fragmentation occurs by a step-wise evaporation of one monomer at a time. In this respect we disagree with previous claims that the fragmentation of the serine octamer occurs via evaporation of dimers ${ }^{1}$ (indeed at $V_{c}=30 \mathrm{~V}$ the main fragmentation corresponds to dimer loss, however at lower voltages monomer loss is the main fragmentation product). At $V_{c}>150 \mathrm{~V}$ we observe fragments of smaller masses, corresponding to fragmentation of the serine molecule itself, which are not shown in the figure. 


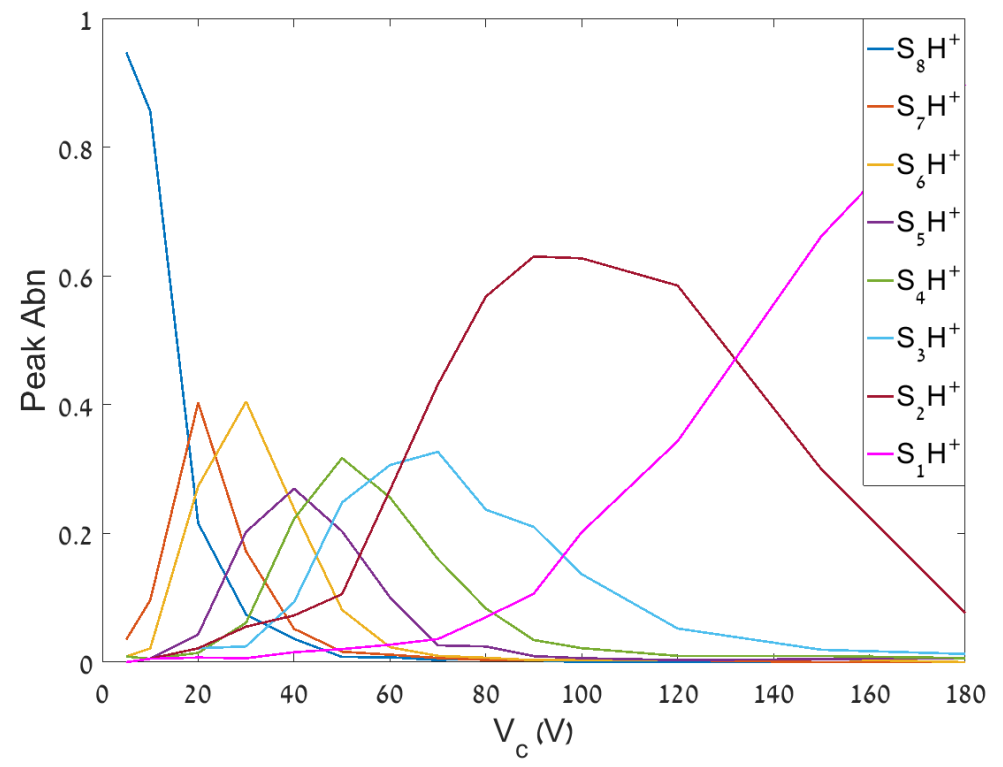

Figure 1: MS/MS results for the fragmentation of the serine octamer as a function of the collision voltage, $V_{c}$.

\section{References}

(1) Cooks, R. G.; Zhang, D.; Koch, K. J.; Gozzo, M. N., Fabio C.and Eberlin Chiroselective Self-Directed Octamerization of Serine: Implications for Homochirogenesis. Anal. Chem. 2001, 73, 3646-3655. 